\title{
Methodology for Optimal Deployment of Corrective Control Measures to Ensure Transient Stability of Uncertain Power Systems \\ DOI:
}

10.1109/TPWRS.2020.3035113

\section{Document Version}

Accepted author manuscript

Link to publication record in Manchester Research Explorer

Citation for published version (APA):

Morales Alvarado, J., \& Milanovic, J. V. (2020). Methodology for Optimal Deployment of Corrective Control Measures to Ensure Transient Stability of Uncertain Power Systems. IEEE Transactions on Power Systems. https://doi.org/10.1109/TPWRS.2020.3035113

\section{Published in:}

IEEE Transactions on Power Systems

\section{Citing this paper}

Please note that where the full-text provided on Manchester Research Explorer is the Author Accepted Manuscript or Proof version this may differ from the final Published version. If citing, it is advised that you check and use the publisher's definitive version.

\section{General rights}

Copyright and moral rights for the publications made accessible in the Research Explorer are retained by the authors and/or other copyright owners and it is a condition of accessing publications that users recognise and abide by the legal requirements associated with these rights.

\section{Takedown policy}

If you believe that this document breaches copyright please refer to the University of Manchester's Takedown Procedures [http://man.ac.uk/04Y6Bo] or contact uml.scholarlycommunications@manchester.ac.uk providing relevant details, so we can investigate your claim.

\section{OPEN ACCESS}




\title{
Methodology for Optimal Deployment of Corrective Control Measures to Ensure Transient Stability of Uncertain Power Systems
}

\author{
Juan D. Morales, Student Member, IEEE, and Jovica V. Milanović, Fellow, IEEE
}

\begin{abstract}
This paper proposes a method for the optimal deployment of Corrective Control Measures (CCMs) for the improvement of transient stability in uncertain power systems. First, the critical oscillation patterns of the disturbed system are identified using Hierarchical Clustering (HC) with statistically defined algorithm parameters. This is followed by the identification of critical generators where the application of CCMs will have maximum impact. The results show that a significant reduction in multi-machine unstable events can be achieved by the strategic deployment of CCMs. The proposed method is demonstrated on a modified IEEE 68 bus NETS-NYPS test network with Renewable Energy Sources (RES) and realistically modelled system uncertainties.
\end{abstract}

Index Terms-Corrective control, hierarchical clustering, multi-machine instability, transient stability improvement.

\section{INTRODUCTION}

$\mathbf{O}$ VER the past few decades, the operation of power systems has been increasingly altered by more restrictive market-driven investments and more lately, by a growing integration of Renewable Energy Sources (RES) of inherent uncertain operation into the portfolio of available generation for energy production. Under this continuous trend, that might result in power systems operating at certain times in more stressed conditions and closer to their stability limits, corrective control for the improvement of system transient stability becomes a viable option to be considered to deal with such potential problems.

There is a range of well-known Corrective Control Measures (CCMs) for the improvement of transient stability $[1,2]$. Most of the past work on the subject though, studied each method individually focusing mainly on the modelling and control of each specific device to maximize its impact on transient stability [3]. Considering that, to a certain extent, most non-disruptive CCMs can be installed at will in large systems, it is clear that a cost-effective stabilization strategy will require an optimal deployment of such devices so that their system-wide impact can be maximized. This however, has not been addressed in a systematic way, if at all, in the

This was supported by the Department of Electrical and Electronic Engineering PhD scholarship award of The University of Manchester and by the EPSRC, U.K., through the HubNet consortium (grant EP/N030028/1).

The authors are with the Department of Electrical and Electronic Engineering, The University of Manchester, Manchester, M60 1QD, U.K. (email: juan.morales@manchester.ac.uk; jovica.milanovic@manchester.ac.uk). past. In [4, 5], coherency was successfully employed for the installation of a reduced number of CCMs, namely Braking Resistors (BRs), to one generator per group of coherent generators. In these studies though, either the exact location of the BRs, their size, or the fault data for finding the groups of coherent generators, were obtained heuristically without developing a consistent methodology.

Clustering algorithms that use rotor angle or speed responses for coherency identification have been reported in [6-9]. In [10], Hierarchical Clustering (HC) is applied for the identification of characteristic oscillation patterns (or dynamic signature) of rotor angle responses obtained by a probabilistic Monte Carlo (MC) approach. An important improvement of $\mathrm{HC}$ with respect to other clustering methods is that the number of clusters does not need to be specified in advance, and they can be automatically identified if a proper threshold for cutting off the hierarchical trees is pre-defined. Nevertheless, since the clustering has to be performed by taking a sample of rotor angles at one point in time during the oscillation, the composition and number of clusters are also a function of the chosen point in time for cluster formation, in addition to the cut-off threshold. In [11-13], HC was studied alongside classification algorithms, as data mining techniques, which can be effectively used for dynamic signature identification, employing different criteria for the time of cluster formation and cut-off threshold parameters, without discussion of their optimal values or how they might influence the resulting grouping patterns. As a matter of fact, results in these studies show that the number of oscillation patterns obtained for a system is usually large, making it difficult to identify critical groups/generators in a straightforward way that could guide a further development of a stabilization strategy by the deployment of CCMs. This is especially the case when dealing with multi-machine instability events which may involve a large number of unstable generators.

This paper proposes a robust method for the optimal deployment of CCMs for the improvement of transient stability in large uncertain power networks. The procedure uses $\mathrm{HC}$ with improved clustering parameters for the grouping of rotor angle responses to find a reduced number of unstable patterns and generators where the application of CCMs will have maximum impact. The main contributions of this paper are: i) The statistical definition of the instant of cluster formation and cut-off threshold values for the grouping and 
identification of critical representative patterns of unstable generators using HC. ii) The development of a stabilization procedure for the optimal deployment of CCMs based on information from the proposed $\mathrm{HC}$ method, aimed at reducing the probability of multi-machine instability. The application of the developed concepts is illustrated on a modified IEEE 68 bus NETS-NYPS test network incorporating RES and realistically modelled system uncertainties.

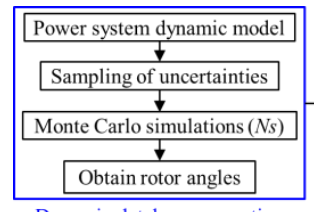

Dynamic database generation

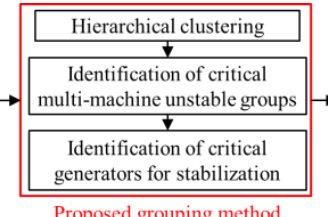

Proposed grouping method

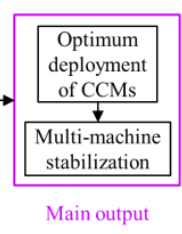

Main output
Fig. 1. Flowchart of the proposed stabilization procedure.

\section{MethodolOGY}

The flowchart shown in Fig. 1 outlines the steps involved in the proposed stabilization procedure. Even though the CCMs operate always after a disturbance, their deployment in the network is a result of studies at the planning stage. Therefore, the database containing system dynamic responses must reflect thoroughly their probabilistic dynamic behavior for an appropriate period of time. The MC approach used in this paper is considered sufficient for producing an adequate number of responses to test the methodology.

\section{A. Generation of System Dynamic Response Database}

The MC approach developed in [12] is used to generate an appropriate number (as discussed in Section III-D) of realistic responses for a system with the inclusion of RES. The sampling of uncertainties is done separately for each relevant random variable of the system and according to appropriate probability distributions describing their particular behavior.

The pre-fault operating conditions are obtained by solving an Optimal Power Flow (OPF) considering system loading and wind/PV generation uncertainties for a 24 hour period. The dispatch obtained from OPF determines the amount of disconnection of conventional generation and corresponding system inertia reduction due to RES penetration. Post-fault dynamic responses are obtained by a Time Domain Simulation (TDS) considering fault location and duration uncertainties. A total number of $N s$ simulations are performed to accurately represent the whole dynamic behavior of the system. Further details about the $N s$ value, system modelling and uncertainties used in the study are provided in Section III.

Rotor angle responses of each generator are used to identify oscillation patterns of the system (system dynamic signature) using the clustering method proposed in this section, as well as to calculate rotor speed and acceleration.

\section{B. Multi-machine Instability Phenomenon}

The concept of electromechanical wave propagation states that when a disturbance occurs, it spreads out to remote points of the system as transient spatial delayed variations of rotor angle and speed oscillations, travelling away from the disturbance location at a finite speed much slower than the speed of light [14-18]. The travelling wave will affect first the closest generators to the disturbance, and may continue to build up when travelling through the system, hitting further generators and possibly causing the loss of synchronism of more than one generator. This may be deemed to be the main mechanism behind the multi-machine instability phenomenon in power systems. Furthermore, if the first group of generators hit by the travelling wave and likely to lose synchronism can be stabilized by strategically located CCMs, the propagation of oscillations will be stopped or at least significantly damped, avoiding further generators to become unstable. It can be expected that this initial group of potentially unstable (or critical) generators will be hit first by the travelling wave within a very short time period. Therefore their oscillations should show some degree of coherency (i.e., their angular separation will be small), at least until an instant after and close to the actual loss of synchronism, if it occurs. Other generators will also oscillate coherently but distinctively apart from the critical group and hence any clustering algorithm could be used to group all of them accordingly. However, the quality of any set of clusters obtained will depend on how well the physical aspects of the rotor angle instability phenomenon can be integrated into the method, on a clear definition of the clustering target, and on the specifics of the algorithm itself, i.e., clustering parameters, amount of data used, etc.

Regardless of the type of method used, if the target is to identify the first group of critical generators hit by the travelling wave, then the clustering must not be performed using data too early after the fault. This is because the angular displacement between the critical generators and the rest of generators may not be enough to guarantee their differentiation, so that they can be effectively clustered. On the other hand, if the clustering is performed too late, using data after a loss of synchronism in the system, when the rotor angle magnitudes have already started increasing erratically, then the clustering results may lack any physical and practical meaning due to the randomness of rotor angle behavior during this period. Therefore, timely clustering using rotor angle data close to the instant of a system loss of synchronism will be able to identify the first group of critical, potentially unstable generators, as well as other relevant generator groups oscillating together in a multi-machine unstable event.

\section{Hierarchical Clustering for Multi-Machine Instability}

In this paper, $\mathrm{HC}$ is chosen as the clustering method, since it has already been used successfully for clustering post-fault rotor oscillations in power systems, e.g. [10-13]. In these studies, it was demonstrated that clustering using just a single sample of rotor angles during the post-fault oscillation is enough to group generators effectively. Particularly in [10], clustering experiments using both a single sample and the complete swing curves samples information (trajectory-based clustering) were performed, concluding that the results were absolutely the same using either of the two approaches (though with different algorithm parameters for each case).

As in [10], the proposed procedure in this paper clusters the rotor angle data by the agglomerative (bottom-up) strategy. It first finds the similarity between a pair of objects (rotor 
angles) and then links sets of objects for merging with the upper levels into a hierarchical tree, i.e., a dendrogram, by means of a predefined linkage criterion. Euclidean distance between data points is used as the similarity measure and complete linkage (distance between clusters equal to the distance between the objects in each cluster that are the farthest away) is chosen as the linkage criterion. The final clusters are determined by the selection of an appropriate cutoff threshold value for the hierarchical tree. Fig. 2 illustrates the clustering procedure. Fig. 2(a) shows a typical post-fault rotor oscillation of six generators and three different instants for clustering the angle data, $t_{1}, t_{2}$ and $t_{3}$. Fig. 2(b) shows the obtained dendrograms for each of the three instants, in addition to different values of cut-off thresholds. It is observed that three clusters (or groups) of coherent generators exist, G1G2, G3-G4 and G5-G6, this resulting arrangement of clusters for this event is called a Grouping Pattern (GP). The cluster containing G1-G2 is the Critical Group (CG) of generators for this GP, since it is first hit by the travelling wave and will likely lose synchronism for that event. It is also observed that for the clustering instant $t_{3}$, a clear identification of this GP can be achieved. However, there is still a wide range where $t_{3}$ can be exactly located where this GP can be readily identified. Moreover, even for a clustering in $t_{3}$, if a proper cut-off threshold value is not defined for the corresponding dendrogram, i.e. between $20-80^{\circ}$ according to Fig. 2(b), this optimal or true GP will still be missed by the algorithm.

It is clearly evidenced that both the instant of clustering and the cut-off threshold values completely control the clustering results, and that relatively slight changes of their values could produce significantly different clusters. It is this aspect of generator clustering that previous work has not studied, nor proposed the criteria for identifying and setting the optimal values of these parameters. In this paper, the optimal estimation of the instant of clustering and the cut-off threshold values are determined using appropriate statistic measures. They assess the appropriateness and quality of cluster partitions over the set of representative rotor angle responses obtained as described in Section II-A. This ensures that the actual critical oscillation patterns of a system are identified.

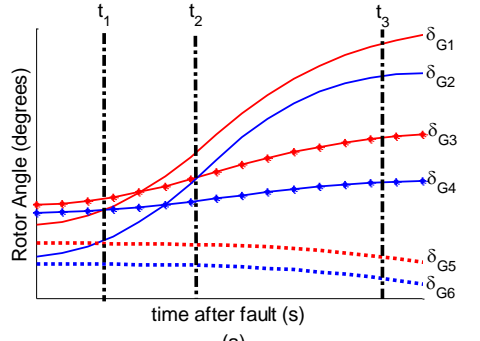

(a)

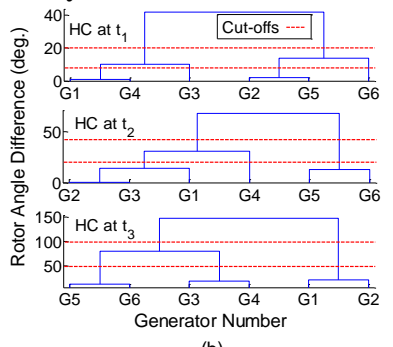

(b)
Fig. 2. (a) Instants for HC sampling and (b) corresponding dendrograms

As previously discussed, the clustering should be done at the instant close to the loss of synchronism. Hence it is better to define this in terms of an angle threshold for instability detection rather than an actual instant of time after a disturbance. There is currently no general agreement on a definitive angle threshold for transient instability detection in multi-machine stability studies. However, in [19], a thorough analysis on the subject was done, concluding that Center Of Inertia (COI) frame based thresholds offer some conceptual and practical advantages compared to approaches measuring the difference between pairs of rotor angles. Therefore, the angle data in the COI frame are used in this paper to determine the samples at which the clustering should be performed, as it gives more insights into how groups of machines separate from each other while approaching their stability limits. The position of the COI is defined by (1).

$$
\delta_{C O I}=\frac{\sum_{i=1}^{N_{G}} H_{i} \times \delta_{i}}{\sum_{i=1}^{N_{G}} H_{i}}
$$

where $H_{i}$ is the inertia constant of generator $i, N_{G}$ is the total number of generators and $\delta_{i}$ is the rotor angle with respect to the reference machine. The system can be deemed unstable if condition (2) is reached.

$$
\left|\delta_{i}-\delta_{\text {CoI }}\right|>\delta_{T}
$$

where $\delta_{T}$ is the transient instability threshold and will also denote from now on the threshold for clustering. According to literature, $\delta_{T}$ is a value usually between $100-180^{\circ}$ [19]. This range is hence chosen for the clustering threshold assessment in this study. With respect to the cut-off threshold range, denoted from now on as $\delta_{C}$, it is typically defined to be between $10-100^{\circ}$. Both thresholds are evaluated in $10^{\circ}$ steps.

\section{Statistical Assessment of Clustering Partitions}

The cut-off threshold in $\mathrm{HC}$ algorithms must check that the elements within each of the formed clusters are more similar among them than to elements in different clusters. This can be considered a special case to the more general problem of determining the most appropriate number of clusters for other clustering algorithms besides the hierarchical ones, since the cut-off threshold also automatically defines the number of clusters. However, there is no definitive method to find such a number or a threshold that is optimal and there is a lack of consensus in this respect. Different results are usually obtained for the same data with different preset thresholds, and some methods will be better suited for a particular application than others, with no method fitting all cases. It is therefore recommended not to depend entirely on a single way for finding the optimal number of clusters [20]. The number of resulting clusters depends on the shape and scale of data distribution as well as on the clustering resolution required [21] and the expected goal to be achieved by the clustering process [22]. This implies that it is possible that data could be partitioned in different number of equally acceptable clusters, hence the inherent characteristics of a dataset partition are subjected to some degree of heuristics. An example of such case with data clustered using $\mathrm{HC}$ is provided in [23]. It can be concluded that, as suggested in [24], subjective criteria based on expert judgement still have a substantial influence on a dataset clustering results.

A thorough comparison of 30 different procedures for choosing the cluster partition and/or number of groups is performed in [25], with some of the top performing ones further compared and analyzed in [20,23]. Though some of those are explicitly or implicitly recommended for $\mathrm{HC}$ 
algorithms, all are concerned with the discovery of the optimal number of clusters and therefore can be generally applied to any clustering algorithm. The common denominator for all these methods is that they always involve some degree of heuristics in their definition. This is in the form of either some empirically defined constant parameter of a proposed statistic, or by having a strong correlation with the used clustering method (which would make them perform better only when the rules for choosing the number of clusters make the same assumptions about the data structure as the clustering method itself [20]). There is a consensus in literature, e.g. [20-23], that most methods are based on the behavior of the change of the defined within-cluster dissimilarity $W_{K}$ as a function of the $K$ number of clusters. Basically, the value of $W_{K}$ will decrease as the number of clusters increases because having more clusters allows to group elements that are more similar to each other. However, past the optimal number of clusters $K^{*}$, any partition will split a natural cluster containing elements already similar enough, and the effect on the decrease of $W_{K}$ will become substantially less than before reaching $K^{*}$, and more marginal as $K$ is increased further. The criterion for finding $K^{*}$ is then to identify this turning point or "elbow" in the plot of $W_{K}$ as a function of $K$. This is still a somewhat heuristic approach, since deciding how substantial the change defining the elbow should be is unclear in some cases and subjected to user's judgement and prior expectation. Two recently proposed statistics to find the optimal number of clusters are the Average Silhouette Width (ASW) [26] and the Gap Statistic (GS) [23].

The ASW, defined in (3), is the average of the silhouette coefficient, denoted by $s\left(\delta_{i}\right)$ and defined in (4), over all the elements in a dataset.

$$
\begin{gathered}
A S W=\frac{1}{N_{G}} \sum_{i=1}^{N_{G}} s\left(\delta_{i}\right) \\
s\left(\delta_{i}\right)=\frac{b\left(\delta_{i}\right)-a\left(\delta_{i}\right)}{\max \left\{a\left(\delta_{i}\right), b\left(\delta_{i}\right)\right\}}
\end{gathered}
$$

with values varying from -1 to 1 . For each element $\delta_{i}$, the value $a\left(\delta_{i}\right)$ is the average distance to all other elements in its cluster, which reflects the cluster compactness, while $b\left(\delta_{i}\right)$ is its minimum average distance to all clusters to which it does not belong, which reflects the degree of cluster separation. Thus, large values of $s\left(\delta_{i}\right)$ indicate that the element is well clustered. The silhouette coefficient was defined as a metric for assessing the quality of clusters. Nevertheless, choosing a partition that maximizes the ASW provides a formal criterion for selecting the optimal number of clusters [20]. In [26], it is stated that a reasonable classification will yield an ASW above 0.5 , while values below 0.2 should be interpreted as a lack of substantial cluster structure. For some clustering algorithms, having fewer elements per cluster could achieve better results [21], considering that the granularity of any dataset would be maximized having one element per cluster. This, however, does not provide any useful partition of the data and might unrealistically increase the value of the ASW, hence it is undesirable.
The GS formalizes the heuristic elbow criterion for estimating the optimal number of clusters to automatically (or statistically) locate when the elbow occurs. The gap curve $G(K)$ is defined in (5).

$$
G(K)=E\left\{\log \left(W_{K}^{*}\right)\right\}-\log \left(W_{K}\right)
$$

where $W_{K}^{*}$ is the within cluster dissimilarity of clustering data from an appropriate null distribution and $E$ represents the mathematical expectation. For the general case of a multivariate dataset having elements with more than one feature (or dimension), a suitable null reference distribution is generated using MC sampling from data uniformly distributed over a box containing the original dataset elements over each feature. It estimates the optimal number of clusters $K^{*}$ to be where the gap curve value is the largest. The formal definition for estimating $K^{*}$ is given in (6).

$$
K^{*}=\underset{K}{\operatorname{argmin}}\left\{K \mid G(K) \geq G(K+1)-s_{K+1}^{\prime}\right\}
$$

where $s_{K}^{\prime}$ is an error term due to the MC sampling [22]. As shown in [23], the GS performs better over a set of simulated data examples than the top performers of the study presented in [25], including the ASW. Since based on the principles of the elbow criterion, some parameters for testing still rely on the user's judgement, i.e., the number of MC samples, the value of the error term $s_{K}^{\prime}$, the choice for generating the reference distribution for the multivariate case, and the choice of $W_{K}$ (squared error, squared distances around cluster means, etc.). On the other hand, for the application to clustering multimachine oscillations, where each element (generator) only has its rotor angle as a feature for clustering (one dimension), Theorem 1 proposed in [23] for the univariate case can be used. It states that for data in one dimension, the uniform distribution is the most likely to produce spurious clusters by means of the GS test. Hence, a single uniform distribution over the data can be used directly for generating the null reference distribution without the need of MC sampling, also removing the error term in (6) and thus further reducing the heuristics involved. Another advantage of the GS, is that if the produced gap curve exhibits non-monotone behavior, a smaller well-separated cluster arrangement (or GP) within a larger well-separated one, i.e., the optimal number of clusters obtained from (6), may exist, implying that more than one natural partition trend may be present in the dataset. In such cases, examination of the entire gap curve is suggested, rather than only finding the position of its maximum.

Both the ASW and GS (using within cluster sum of squares as $W_{K}$ and $\left.K^{*}>1\right)$ are chosen in this paper to assess statistically the rotor angles clustering arrangements or GPs. On one hand, the ASW metric properties will be useful when comparing the quality of the GPs obtained from different clustering parameters. On the other, the GS test results will serve as a benchmark allowing filtering out GPs that do not present the optimal number of clusters, so that a quality comparison between only valid GPs that passed the test can be performed. Besides, it has been observed in the past that the GS has less heuristics involved in estimating the number of clusters. 


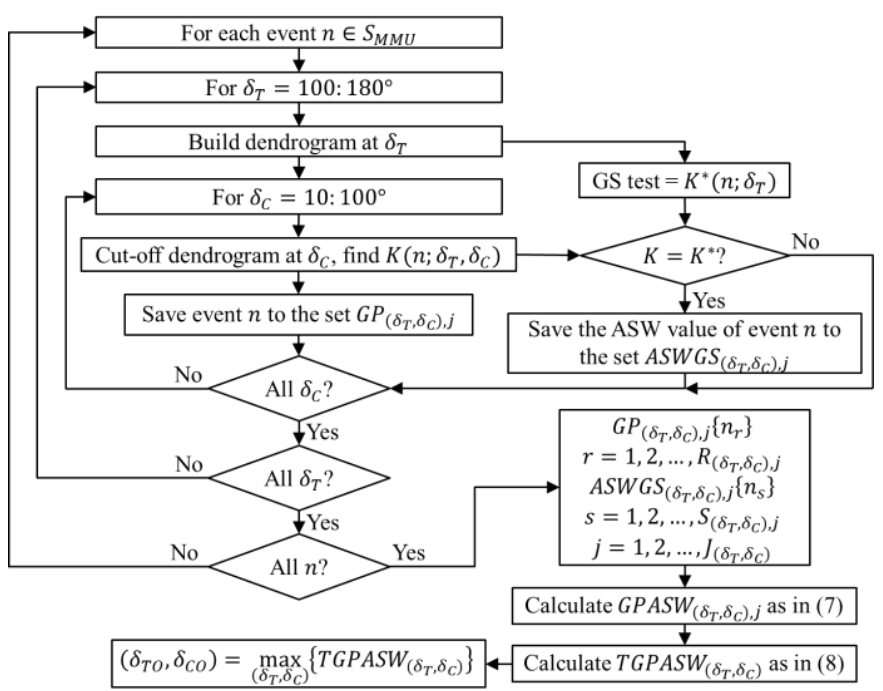

Fig. 3. Flowchart of the statistical clustering assessment.

The proposed clustering assessment is described in the flowchart of Fig. 3. Where:

$S_{M M U}$ : set of all fault events resulting in multimachine instability.

$n_{M M U}$ : number of elements of $S_{M M U}$.

$\left(\delta_{T}, \delta_{C}\right)$ : clustering parameters, for $\delta_{T}=\left[100^{\circ}: 180^{\circ}\right]$ and $\delta_{C}=\left[10^{\circ}: 100^{\circ}\right]$ (both in $10^{\circ}$ steps).

$K^{*}\left(n ; \delta_{T}\right)$ : optimal number of clusters found for event $n$ using the GS test, for clustering at $\delta_{T}$.

$K\left(n ; \delta_{T}, \delta_{C}\right)$ : number of clusters for event $n$ obtained with a cut-off value $\delta_{C}$, for clustering at $\delta_{T}$.

$J_{\left(\delta_{T}, \delta_{C}\right)}$ : number of GPs produced with parameters $\left(\delta_{T}, \delta_{C}\right)$ for all events that belong to $S_{M M U}$.

$G P_{\left(\delta_{T}, \delta_{C}\right), j}$ : set containing all grouped events that belong to the $j^{\text {th }}$ GP obtained with clustering parameters $\left(\delta_{T}, \delta_{C}\right) . j=\left\{1,2, \ldots, J_{\left(\delta_{T}, \delta_{C}\right)}\right\}$.

$R_{\left(\delta_{T}, \delta_{C}\right), j}$ : number of elements (events) contained in each set $G P_{\left(\delta_{T}, \delta_{C}\right), j} \cdot \sum_{\forall j} R_{\left(\delta_{T}, \delta_{C}\right), j}=n_{M M U}$.

$\operatorname{ASWGS}_{\left(\delta_{T}, \delta_{C}\right), j}$ : set containing grouped ASW values only of events with GPs having $K=K^{*}$ clusters according to the GS test and belonging to the $j^{\text {th }}$ GP obtained with clustering parameters $\left(\delta_{T}, \delta_{C}\right) . j=\left\{1,2, \ldots, J_{\left(\delta_{T}, \delta_{C}\right)}\right\}$.

$S_{\left(\delta_{T}, \delta_{C}\right), j}$ : number of elements contained in each set $\operatorname{ASWGS}_{\left(\delta_{T}, \delta_{C}\right), j}\left(S_{\left(\delta_{T}, \delta_{C}\right), j} \leq R_{\left(\delta_{T}, \delta_{C}\right), j}\right)$.

Therefore, a search over all the predefined values for the instants for clustering $\delta_{T}$ and cut-off thresholds $\delta_{C}$ is performed for all the events from the set of multi-machine unstable events for a given system. Each pair $\left(\delta_{T}, \delta_{C}\right)$ will produce a number of $J_{\left(\delta_{T}, \delta_{C}\right)}$ GPs, each of which consists of a certain number of $R_{\left(\delta_{T}, \delta_{C}\right), j}$ events, which are grouped into the sets $G P_{\left(\delta_{T}, \delta_{C}\right), j}$, with $j=1,2 \ldots, J_{\left(\delta_{T}, \delta_{C}\right)}$. In parallel, for each event $n$ and instant of clustering $\delta_{T}$, a GS test is performed, yielding an estimation of the optimal number of clusters $K^{*}\left(n ; \delta_{T}\right)$. This result is compared against the number of clusters $K\left(n ; \delta_{T}, \delta_{C}\right)$ obtained directly for each of the predefined cut-off threshold values $\delta_{C}$, then only if $K=K^{*}$, the ASW of the event's GP is calculated and stored in the set $\operatorname{ASWG} S_{\left(\delta_{T}, \delta_{C}\right), j}$, each one finally having a total of $S_{\left(\delta_{T}, \delta_{C}\right), j}$ elements. With this information, a quality measure can be assigned to all GPs according to (7).

$$
\operatorname{GPASW}_{\left(\delta_{T}, \delta_{C}\right), j}=\frac{1}{R_{\left(\delta_{T}, \delta_{C}\right), j}} \sum_{s=1}^{S_{\left(\delta_{T}, \delta_{C}\right), j}} \operatorname{ASWGS}_{\left(\delta_{T}, \delta_{C}\right), j}\left\{n_{S}\right\}
$$

where $n_{s}$ is the calculated ASW value of each element (event) of the set $A S W G S_{\left(\delta_{T}, \delta_{C}\right), j}$, for all $j=1,2 \ldots, J_{\left(\delta_{T}, \delta_{C}\right)}$. The

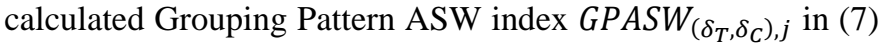
is an ASW type metric but averaged over the $R_{\left(\delta_{T}, \delta_{C}\right), j}$ elements (events) of the $G P_{\left(\delta_{T}, \delta_{C}\right), j}$ sets instead of over the total $S_{\left(\delta_{T}, \delta_{C}\right), j}$ elements (events) of the $A S W G S_{\left(\delta_{T}, \delta_{C}\right), j}$ sets themselves (with $S_{\left(\delta_{T}, \delta_{C}\right), j} \leq R_{\left(\delta_{T}, \delta_{C}\right), j}$ ). In this way, the $G P A S W_{\left(\delta_{T}, \delta_{C}\right), j}$ index represents a quality measure that takes into account only validated clustered events whose GPs were correctly estimated according to the GS test, and as such, it quantifies the degree to which that identified GP represents an optimal or true oscillation pattern for the system. In order to compare the $\mathrm{HC}$ results obtained from all pairs of clustering parameters $\left(\delta_{T}, \delta_{C}\right)$, a total quality measure is defined in (8).

$$
\operatorname{TGPASW}_{\left(\delta_{T}, \delta_{C}\right)}=\frac{1}{J_{\left(\delta_{T}, \delta_{C}\right)}} \sum_{j=1}^{J_{\left(\delta_{T}, \delta_{C}\right)}} \operatorname{GPASW}_{\left(\delta_{T}, \delta_{C}\right), j}
$$

where the Total Grouping Pattern ASW index $T G P A S W_{\left(\delta_{T}, \delta_{C}\right)}$ is the simple average of the $G P A S W_{\left(\delta_{T}, \delta_{C}\right), j}$ values, since each of the $J_{\left(\delta_{T}, \delta_{C}\right)}$ obtained GPs would represent a true oscillation pattern according to the clustering parameters $\left(\delta_{T}, \delta_{C}\right)$, and hence must be assigned the same level of importance.

In a similar way to the use of the maximum ASW value as a means to determine the best clustering partition of a dataset, the maximum value of the proposed $\operatorname{TGPASW}_{\left(\delta_{T}, \delta_{C}\right)}$ index over all clustering parameter pairs $\left(\delta_{T}, \delta_{C}\right)$ will yield the optimal or true set of GPs that best represent the multimachine unstable oscillation patterns for any power system. Therefore, the optimal clustering parameters $\left(\delta_{T O}, \delta_{C O}\right)$ of the $\mathrm{HC}$ algorithm as proposed in this study can be found using (9).

$$
\left(\delta_{T O}, \delta_{C O}\right)=\max _{\left(\delta_{T}, \delta_{C}\right)}\left\{T G P A S W_{\left(\delta_{T}, \delta_{C}\right)}\right\}
$$

\section{E. Proposed Stabilization Procedure}

Each of the total $J_{\left(\delta_{T O}, \delta_{C O}\right)}$ optimal GPs sets $G P_{\left(\delta_{T O}, \delta_{C O}\right), j}$ obtained by finding the maximum TGPASW index contains a subset comprising the Critical Group (CG) of generators as defined in Section II-B (denoted as $C G_{j}$ ), i.e., the group or cluster hit first by the travelling wave (cluster of G1-G2 in the illustrative example of Fig. 2 in Section II-B). Each of the GPs will be stabilized independently by finding their most unstable event, called the Critical Event (CEV), among all $R_{\left(\delta_{T O}, \delta_{C O}\right), j}$ events from each $G P_{\left(\delta_{T O}, \delta_{C O}\right), j}$ set, and the Critical Generator (CGEN), which belongs to the subset $C G_{j}$. This straightforward systematic procedure can be devised since it can be expected that if the CEV per GP can be stabilized by appropriate CCMs, then all other events of the same group will be also stabilized as they are less critical. It is also expected that only one CGEN from the subset $C G_{j}$ is able to 
control each multi-machine oscillation pattern, considering that the critical right ones have been identified, as it is the case with the proposed methodology. The identification of the CEV can be done by using an appropriate transient stability index that can measure the severity of all events for each GP and rank them accordingly. For the present study, the Average Acceleration Index $A A I_{j}\left(n_{r}\right)$ per event $n_{r}$ and for each $G P_{\left(\delta_{T O}, \delta_{C O}\right), j}$ as defined in (10), with $r=1,2, \ldots, R_{\left(\delta_{T O}, \delta_{C O}\right), j}$ and $j=1,2, \ldots, J_{\left(\delta_{T O}, \delta_{C O}\right)}$, is used to make up the ranking.

$$
A A I_{j}\left(n_{r}\right)=\frac{1}{T_{r}-T_{0}} \cdot \int_{T_{0}}^{T_{r}} a_{C G E N_{j}} d t
$$

where $a_{C G E N_{j}}$ is the rotor acceleration of the $C G E N_{j}$ for each $C G_{j}\left(\mathrm{rad} / \mathrm{s}^{2}\right), T_{0}$ is the instant of fault and $T_{r}$ is the instant of clustering when the first generator reaches $\delta_{T O}$ for event $n_{r}$ (both in seconds). The value of $T_{r}$ is different for each $n_{r}$ since the angle threshold for clustering $\delta_{T O}$ is reached during the oscillation at different instants of time for each event. The $\operatorname{CGEN}_{j}(12)$ is the one having the maximum $A A I_{j}\left(G_{i}\right)$ sum for all the events $R_{\left(\delta_{T O}, \delta_{C O}\right), j}$ of a GP as defined in (11).

$$
\begin{gathered}
A A I_{j}\left(G_{i}\right)=\sum_{r=1}^{R_{\left(\delta_{T O}, \delta_{C O}\right), j}}\left(\frac{1}{T_{r}-T_{0}} \int_{T_{0}}^{T_{r}} a_{i r} d t\right), \forall G_{i} \in C G_{j} \\
C G E N_{j}=\max _{G_{i}} A A I_{j}\left(G_{i}\right), \forall j
\end{gathered}
$$

where $a_{i r}$ is the acceleration of generator $G_{i}$ belonging to $C G_{j}$ during event $n_{r}$ for $j=1,2, \ldots, J_{\left(\delta_{T O}, \delta_{C O}\right)}$. Since the stabilization of the CGENs obtained by (12) would avoid the multi-machine instability, the CCMs must be applied directly to them, or their corresponding $\mathrm{LV}$, or $\mathrm{HV}$ buses, as the impact of CCMs is maximized there and will be reduced if applied or installed to a farther bus or other locations in the system. I.e., the identification of the CGENs automatically determines the location where CCMs should be applied irrespectively of the type of CCM. By defining the calculation of the acceleration indices as in (10) and (11) until the instant of clustering $T_{r}$, which is close to the stability limit and happens after a fault is cleared, not only the criticality of the fault itself is considered but also the capability of the system to decelerate the rotors after the fault clearing.

The stabilization procedure is summarized as follows: i) identify the CEV and the CGEN by (10) and (12), respectively, independently for each $j^{\text {th }}$ of the GPs; ii) determine the amount/size of the selected CCM to be applied only to the $C G E N_{j}$ to stabilize only the $C E V_{j}$ (e.g. MVAR if reactive compensation based CCMs are used, MWs if BRs, load or generation shedding are used, etc.); iii) test the effectiveness of the CCM determined in ii) by simulating again the remaining events of each GP; iv) if all the remaining events are not stabilized, identify a new CEV among the ones that were not stabilized using (10) with the CCM in place and return to step ii); v) repeat this process until full stabilization is achieved; vi) with the deployed CCMs obtained for each GP, a final set of CCMs is defined based on the resulting reduction of the probability of instability obtained per group with respect to the case without any CCM.

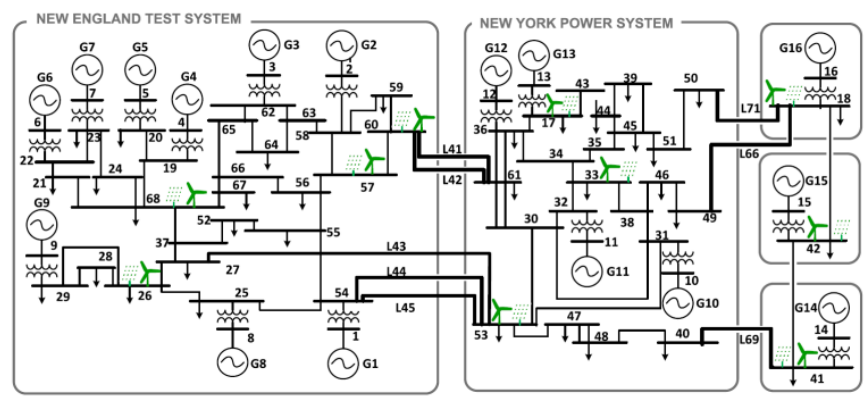

Fig. 4. Modified IEEE bus test network with the inclusion of RES.

\section{CASE StUdy}

\section{A. Test Network}

The test system studied is a modified version of the IEEE 68 bus test network, which is a reduced order equivalent model of the New England Test System (NETS, G1-G9), New York Power System (NYPS, G10-G13), and three additional external systems (G14/G15/G16). Complete data of the conventional part of the network can be found in [27]-[28]. Synchronous generators are represented by $6^{\text {th }}$ order dynamic models including generic governors and slow DC exciters (IEEE-DC1A), except for G9 which is equipped with a fast static exciter (IEEE-ST1A) and a Power System Stabilizer (PSS). RES plants at each bus, located as shown in Fig. 4, comprise $66.67 \%$ of Doubly Fed Induction Generators (DFIGs) representing wind generation, and $33.33 \%$ of Full Converter Connected (FCC) units representing both wind and Photo-Voltaic (PV) units (30\% and 70\%, respectively). DFIG and FCC units are represented by generic Type 3 and Type 4 models, respectively [29, 30], suitable for large scale stability studies. All RES modelled have Fault Ride Through (FRT) capabilities and remain connected during a fault.

\section{B. Sampling of Uncertainties}

Typical 24 hour loading and PV curves data are obtained from National Grid [31] and [32], respectively. The system loads are modelled as constant impedances. The hour of the day is sampled randomly using a uniform distribution. In addition, uncertainties for every hour are considered by using a normal distribution for the system loads with a mean value of 1 p.u. and $3 \sigma=10 \%$ [11], and a Beta distribution for the PV generation [33] with $a$ and $b$ parameters equal to 13.7 and 1.3, respectively [34]. For the wind generation, the uncertainty of the wind speed is modelled using a Weibull distribution with parameters $\varphi=11.1$ and $k=2.2$ [35], and the value obtained is considered to be constant throughout the day [36], then the power curve of a typical wind generator is used to derive the power output [37]. All distributions are sampled separately for each parameter in the system so that the variability of the uncertainties is represented in a realistic manner.

Only three phase self-clearing faults are considered. The locations of faults along each line and the line itself are sampled with a uniform distribution. A normal distribution with a mean value of 14 cycles and $3 \sigma=20 \%$ is used to model the fault duration. A longer than typical (approx. 100ms) fault duration is selected to generate an appropriate number of stable/unstable cases for testing the methodology [11]. 


\section{RES and Conventional Generation Disconnection}

The case study presented in this paper has a RES nominal installed capacity (penetration level) of $20 \%$ from the total system conventional generation capacity (in MW). The actual amount of power produced by the RES generation will depend on their resulting sampled uncertainties, and then an OPF, with cost functions adopted from [38], will determine the final conventional generation dispatch, a reduced one compared to a case without RES in the network. When the magnitude of this reduced dispatch involves the disconnection of some generator units in a power plant, the system inertia decreases in discrete amounts according to the nominal capacity of the disconnected generators, since the system operating inertia is directly proportional to the system operating nominal MVA installed capacity of all generators, regardless of their dispatch. Therefore, by quantifying the amount of the total operating installed capacity with respect to the total installed capacity of the system, in MVA, the percentage of inertia reduction in the system is also automatically defined.

In this study, each plant is modelled as a single equivalent generator, and is conceived as comprising an infinite number of units, so that their operating installed capacity $M V A_{G_{i}}$ can be calculated continuously, instead of discreetly, directly from the MW dispatch results of the OPF, using (13).

$$
M V A_{G_{i}}=\frac{P_{G_{i}}}{\left(1-\operatorname{SCap}_{G_{i}}\right) \times N p f_{G_{i}}}
$$

where $P_{G_{i}}$ is the generator dispatch obtained from OPF (MW), $S C a p_{G_{i}}$ is the spare capacity or reserve (p.u.), and $N p f_{G_{i}}$ is the nominal power factor of the generator. For the present study, a fixed $15 \%$ of $S_{C a p}$ is considered as a general dispatch criterion, with all generators having a $N p f_{G_{i}}$ of 0.85 . In case the resulting $M V A_{G_{i}}$ is larger than the nominal MVA installed capacity of a generator, it is set to that nominal value, the reserve is reduced accordingly, and no conventional generation disconnection takes place. If the resulting $M V A_{G_{i}}$ is smaller than the nominal MVA installed capacity of a generator, which is mostly the case due to the presence of RES, then the operating installed capacity of that generator is reduced to $M V A_{G_{i}}$. The percentage of system inertia reduction INRED for that operating condition can be calculated by (14).

$$
I N R E D=\frac{\sum_{i}^{N_{G}} N M V A_{G_{i}}-\sum_{i}^{N_{G}} M V A_{G_{i}}}{\sum_{i}^{N_{G}} N M V A_{G_{i}}} \times 100(\%)
$$

where $N M V A_{G_{i}}$ is the nominal MVA installed capacity of $G_{i}$.

\section{Monte Carlo Simulations}

Eq. (15) gives the estimated relative error $\varepsilon_{r}$ as a function of the number of MC simulations $N_{S}$, as proposed in [39].

$$
\varepsilon_{r}=\frac{\Phi^{-1}\left(1-\frac{\alpha}{2}\right) \times \sqrt{\sigma_{S}^{2}(X) / N_{S}}}{E_{S}(X)}
$$

where $\Phi^{-1}$ is the inverse of the normal standard Cumulative Distribution Function (CDF), $\alpha$ is the confidence level, $\sigma_{S}{ }^{2}$ is the sample variance, $E_{S}$ is the sample mean, and $X$ is the random variable of measured output samples obtained in each MC simulation that wants to be estimated. The criterion for choosing the value of $N_{S}$ is that the $\varepsilon_{r}$ is below $5 \%$ with a $99 \%$ coverage probability $(\alpha=0.01)$. As in [12], the Transient Stability Index (TSI) is defined as the random variable. It is found that by choosing $N_{S}=10,000[19,40]$, for the study case presented in this paper, $\varepsilon_{r} \approx 2.62 \%$, which is lower than the set threshold of $5 \%$, hence exceeding the required accuracy (the set threshold of $\varepsilon_{r} \approx 5 \%$ was actually achieved with less than 6,000 simulations). Each MC simulation involves a TDS of the system subjected to a three phase fault. The simulation time was limited to $5 \mathrm{~s}$, with the fault applied at $1 \mathrm{~s}$, since the analysis focuses on first swing instability. All simulations are performed in the DigSILENT - Power Factory software.

\section{E. Transient Stability Assessment and Generator Clustering}

The MC assessment of the testing scenario results in 925 $(9.3 \%)$ unstable cases out of 10,000 simulated faults. From the 925 unstable cases, $701(76 \%)$ are single-machine and 224 $(24 \%)$ are multi-machine unstable cases. Only the latter are further analyzed as proposed in Section II-D. The main results are illustrated using the surface plots of Fig. 5 as a function of the clustering parameters $\left(\delta_{T}, \delta_{C}\right)$. Fig. 5(a) shows the total ASW and Fig. 5(b) the total number $J_{\left(\delta_{T}, \delta_{C}\right)}$ of GPs. It can be seen that all values of the ASW are greater than 0.62 , indicating that the data does present classifiable trends over the entire clustering predefined region. For low values of $\delta_{C}$, the ASW is the highest, however, in this region, $J_{\left(\delta_{T}, \delta_{C}\right)}$ is also the highest and very large (around 100-160), see Fig. 5(b). The ASW values are therefore artificially increased there, as discussed in Section II-D (a very high number of GPs does not provide any useful partition about a dataset). For higher values of $\delta_{C}$, the number of GPs reduces considerably, which is not the case for the ASW, whose values decrease only to a certain extent, showing an increasing trend over the region $\delta_{C}=50$ $90^{\circ}$ and $\delta_{T}=120-150^{\circ}$, as observed in Fig. 5(a). In this area, the $J_{\left(\delta_{T}, \delta_{C}\right)}$ values are less than 20, see Fig 5(b), which suggests that a better clustering might be possible in such region, however it is impossible to draw a definitive conclusion based only on the ASW and $J_{\left(\delta_{T}, \delta_{C}\right)}$ information. More insights are provided in Fig. 5(c), which shows the percentage of events whose GPs coincide with the results of the GS test, i.e. the accuracy. It can be seen that high values of clustering accuracy (70-80\%) are obtained only for the region $\delta_{C}=50-80^{\circ}$ and $\delta_{T}=130-150^{\circ}$, which is in agreement with the trend observed in the ASW values in Fig. 5(a). Finally, Fig. 5(d) shows the TGPASW index values allowing a better assessment of the actual quality of different clustering parameters using the combined properties of the ASW and GS. It is shown, with marked difference, that the optimal clustering parameters displaying the maximum TGPASW value are $\left(\delta_{T O}, \delta_{C O}\right)=\left(130^{\circ}, 70^{\circ}\right)$. The six GPs obtained with these parameters are shown in Table I, where the subscript $\left(\delta_{T O}, \delta_{C O}\right)$ has been dropped from the notation of the first two column headers, indicating also the cluster composition per GP and the CGs, which are underlined.

By comparing results in Table I with parameters $\left(\delta_{T O}, \delta_{C O}\right)$ obtained directly from the TGPASW values shown in Fig. $5(\mathrm{~d})$, with others obtained with $\left(\delta_{T}, \delta_{C}\right)$ pairs in the region 
also showing high clustering accuracy according to Fig. 5(c), it can be seen that the results in Table I present the minimum number of patterns and the minimum number of clusters per pattern ( 3 for each $G P_{j}$ ). This makes them the best ones fully discriminating the unstable oscillation patterns having fewer groups.

Further comparisons of the proposed HC approach are performed against three cases: i) clustering at the last instant of simulation with a predefined cut-off of $360^{\circ}$ as in $[10,11]$. ii) Clustering heuristically at an angle threshold of $\delta_{T}=360^{\circ}$, but measured in terms of the maximum angle difference between any two generators, typically used in the literature [19], with a cut-off also of $\delta_{C}=360^{\circ}$. iii) Clustering as in ii) but for the best cut-off value found among the range $\left[10^{\circ}: 360^{\circ}\right]$ in $10^{\circ}$ steps according to the TGPASW, which results in $\delta_{C}=190^{\circ}$. Results are shown in Table II. Case i) presents a high number of GPs with the lowest quality measures. Since the clustering is done at a point where the angles are already out of step, randomly oscillating, they do

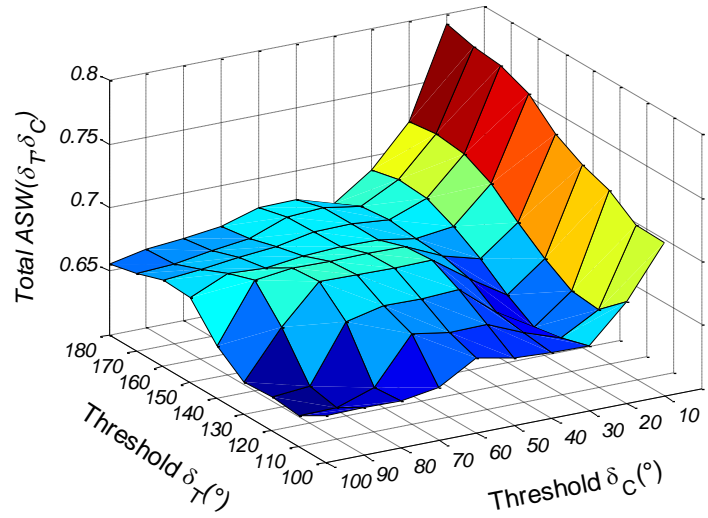

(a)

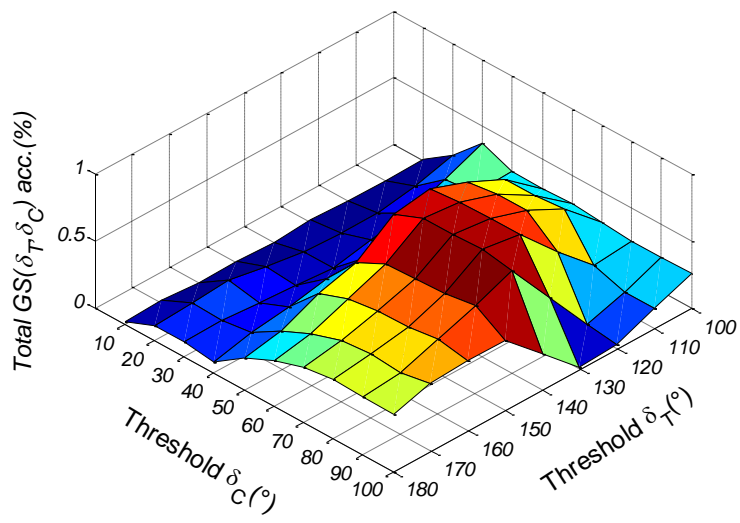

(c) not present a useful pattern that can be discovered. Case ii), using typical instability thresholds, yields a reduced number of 8 GPs, however, these do not represent the data properly, since their quality measures are very low compared to the obtained with the proposed solution. Hence, obtaining a reduced number of GPs is not a definitive indication of a proper clustering of the data. Case iii) shows that even by finding the best cut-off for the typical $\delta_{T}=360^{\circ}$, results are still very far from the proposed method in terms of the quality measures, and with a considerable high number of 18 GPs against the 6 found with the optimal parameters. Besides, the found $\delta_{C}=$ $190^{\circ}$ does not provide any physical insight into the coherency phenomenon, while the non-heuristically found $\left(\delta_{T O}, \delta_{C O}\right)=$ $\left(130^{\circ}, 70^{\circ}\right)$, using the COI frame for measuring rotor angles, does physically offer intuitive thresholds that can be used as a reference for coherency identification as generators start to shift away from each other. All the described features are an indication of the optimality of the obtained results, and will facilitate further steps for the stabilization of the system.

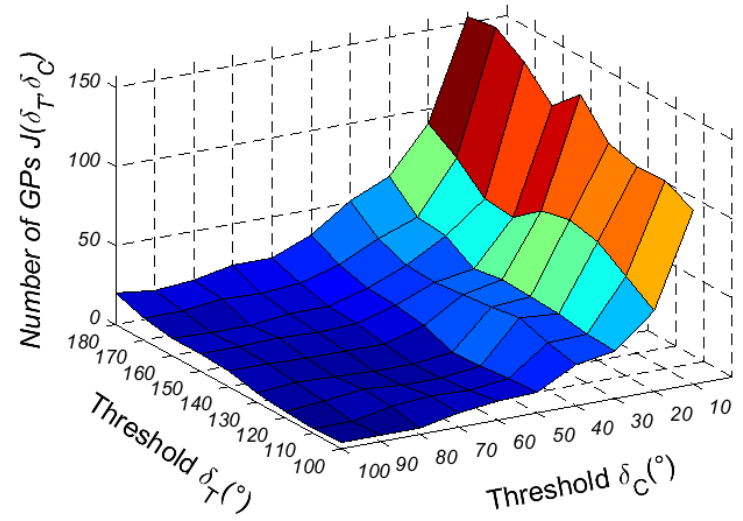

(b)

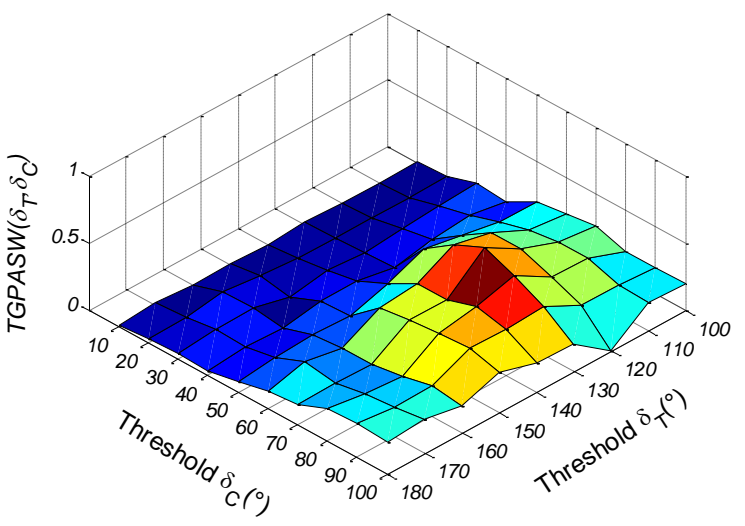

(d)

Fig. 5. Results of the statistical clustering assessment over the entire clustering parameters' region. (a) Average Silhouette Width - ASW. (b) Number of Grouping Patterns (GPs). (c) Percentage of cases that passed the GS test (accuracy). (d) Proposed Total Grouping Pattern ASW - TGPASW index.

TABLE I

OPtimal Multi-Machine UnSTABLE GROUPING PATTERnS - GPS

\begin{tabular}{cccc}
\hline \hline$G P_{j}$ & $R_{j}$ & Freq. & GP's Cluster Composition (CGs underlined) \\
\hline 1 & 113 & $50.4 \%$ & $\underline{(\mathrm{G} 4-\mathrm{G} 5)(\mathrm{G} 2-\mathrm{G} 3 / \mathrm{G} 6-\mathrm{G} 11)(\mathrm{G} 1 / \mathrm{G} 12-\mathrm{G} 16)}$ \\
2 & 74 & $33.0 \%$ & $\underline{(\mathrm{G} 4-\mathrm{G} 7)(\mathrm{G} 2-\mathrm{G} 3 / \mathrm{G} 8-\mathrm{G} 11)(\mathrm{G} 1 / \mathrm{G} 12-\mathrm{G} 16)}$ \\
3 & 17 & $7.6 \%$ & $(\mathrm{G} 6-\mathrm{G} 7)(\mathrm{G} 2-\mathrm{G} 5 / \mathrm{G} 8-\mathrm{G} 11)(\mathrm{G} 1 / \mathrm{G} 12-\mathrm{G} 16)$ \\
4 & 16 & $7.1 \%$ & $\underline{(\mathrm{G} 4-\mathrm{G} 7 / \mathrm{G} 9)(\mathrm{G} 2-\mathrm{G} 3 / \mathrm{G} 8 / \mathrm{G} 10-\mathrm{G} 11)(\mathrm{G} 1 / \mathrm{G} 12-\mathrm{G} 16)}$ \\
5 & 3 & $1.3 \%$ & $\underline{(\mathrm{G} 4 / \mathrm{G} 6-\mathrm{G} 7)(\mathrm{G} 2-\mathrm{G} 3 / \mathrm{G} 5 / \mathrm{G} 8-\mathrm{G} 11)(\mathrm{G} 1 / \mathrm{G} 12-\mathrm{G} 16)}$ \\
6 & 1 & $0.4 \%$ & $\underline{\underline{(\mathrm{G} 6-\mathrm{G} 7)(\mathrm{G} 2-\mathrm{G} 5 / \mathrm{G} 8-\mathrm{G} 11 / \mathrm{G} 16)(\mathrm{G} 1 / \mathrm{G} 12-\mathrm{G} 15)}}$ \\
\hline \hline
\end{tabular}

TABLE II

COMPARISON BETWEEN DIFFERENT HC APPROACHES

\begin{tabular}{cccc}
\hline \hline HC parameters & $\mathrm{N}^{\circ}$ of GPs & GS Acc. & TGPASW \\
\hline Proposed: statistical $\left(\delta_{T O}, \delta_{C O}\right)$ & 6 & $76.34 \%$ & 0.5569 \\
i) Heuristic as in $[10,11]$ & 69 & $15.18 \%$ & 0.1482 \\
ii) Heur. with $\delta_{T}=360^{\circ}, \delta_{C}=360^{\circ}$ & 8 & $27.23 \%$ & 0.1464 \\
iii) Heur. with $\delta_{T}=360^{\circ}, \delta_{C}=190^{\circ}$ & 18 & $34.38 \%$ & 0.3056 \\
\hline \hline
\end{tabular}




\section{F. Deployment of Corrective Control Measures}

The system is stabilized in this study, as an illustrative example of the methodology only, by using Switched Shunt Capacitors (SSHCs) as the CCM. The criteria for the selection of the specific CCM or the associated cost analysis are out of the scope of this study. The main objective of this section is to illustrate the proposed method for CCMs deployment, while the stabilization as such can be performed with any type of CCM available. SSHCs are selected as it was demonstrated in [3] that they perform better when considering non-disruptive CCMs for transient stability improvement compared to more conventional BR devices. Their impact on stability is to some extent comparable to that of Switched Series Compensation (SSC) devices with the advantage of avoiding any subsynchronous resonance issues. The SSHCs are modelled as in [3] and connected to the HV bus $(230 \mathrm{kV})$ of the CGENs.

Results of the procedure described in Section II-E are shown in Table III. All GPs were independently stabilized applying the SSHCs only to the CGENs found by (12) in one iteration (except for CGENs of GPs 2 and 3, which required two iterations), based on the ranking of CEVs obtained using (10). This demonstrates the suitability of the $A A I$ index proposed in this paper and defined by (10) and (11). G5 and G6, with nominal installed capacity of 700 and 1000 MVA, respectively (as shown in Table III), are identified as the CGENs in the test system from the multi-machine transient instability point of view. Therefore, they also represent the optimal locations for deployment of CCMs for transient stability improvement. It can be seen from Table III, that a 1000 MVAR SSHC installed at (close to) G5 and a 600 MVAR SSHC installed close to G6, will guarantee a $100 \%$ stabilization of all 6 GPs. Simulations carried out with the SSHCs having the same MVAR rating but deployed at locations of other generators, not the critical G5 and G6, result in stabilization levels that are lower than $100 \%$, hence demonstrating the optimality of the proposed solution. The results of these simulations are not included here due to space limitations.

TABLE III

INDEPENDENT STABILIZATION RESULTS OF EACH GP's CRITICAL GROUP(CG)

\begin{tabular}{ccccc}
\hline \hline CG & CGEN & $\begin{array}{c}C G E N_{j} \\
\text { (Nom. } \\
\text { MVA) }\end{array}$ & $\begin{array}{c}\text { SSHC size at } \\
C G E N_{j} \text { (MVAR) } \\
1^{\text {st }} \text { iter. / 2 }\end{array}$ & $\begin{array}{l}\text { Cases } \text { iter. } \\
1^{\text {st }} \text { iter. / 2 }\end{array}$ \\
\hline 1 & G5 & 700 & 1000 & $100 \%(113 / 113)$ \\
2 & G5 & 700 & $500 / 1000$ & $95 \%(70 / 74) / 100 \%(74 / 74)$ \\
3 & G6 & 1000 & $200 / 600$ & $82 \%(14 / 17) / 100 \%(17 / 17)$ \\
4 & G5 & 700 & 700 & $100 \%(16 / 16)$ \\
5 & G6 & 1000 & 500 & $100 \%(3 / 3)$ \\
6 & G6 & 1000 & 300 & $100 \%(1 / 1)$ \\
\hline \hline
\end{tabular}

Table IV shows results for 4 sets of CCMs sizes operating simultaneously at the two identified optimal locations, i.e., the CGENs G5 and G6, based on the results in Table III. Table IV also shows the percentage of compensation level measured as the ratio between the SSHC size (in MVAR) and the nominal installed capacity of the corresponding plants (in MVA). It is confirmed that the Set 1 of CCMs is capable of stabilizing the $100 \%$ of the multi-machine unstable events as expected, since the CCMs sizes are defined directly from the results in Table III for a 100\% stabilization of each GP. Set 2 achieves a very high stabilization of $99 \%$ with a total of 400 MVARs less than the critical Set 1. For Set 4 that has the minimum requirements in terms of SSHC sizes, a very acceptable $92.86 \%$ of stabilization is achieved, with nominal SSHCs of 500 and 300 MVAR connected to both CGENs G5 and G6, respectively. These sizes are about $71 \%$ and $30 \%$ of the nominal MVA capacity of the corresponding generators, they also are within magnitudes of compensation already in use in conventional systems, demonstrating the feasibility of the proposed methodology for application in realistic scenarios.

TABLE IV

Total STABILIZATION RESUlTS FOR DifFERENT SETS OF SSHCS SizeS OPERATING SimultaneOUSLY AT THE OPTIMAL Locations G5 AND G6

\begin{tabular}{cccccc}
\hline \hline $\begin{array}{c}\text { Set } \\
\mathrm{N}^{\circ}\end{array}$ & $\begin{array}{c}\text { SSHC } \\
\text { size - G5 } \\
\text { (MVAR) }\end{array}$ & $\begin{array}{c}\text { Comp. } \\
\text { Level } \\
\text { G5 }(\%)\end{array}$ & $\begin{array}{c}\text { SSHC } \\
\text { size - G6 } \\
\text { (MVAR) }\end{array}$ & $\begin{array}{c}\text { Comp. } \\
\text { Level } \\
\text { G6 }(\%)\end{array}$ & Cases Stabilized \\
\hline 1 & 1000 & $143 \%$ & 600 & $60 \%$ & $100 \%(224 / 224)$ \\
2 & 700 & $100 \%$ & 500 & $50 \%$ & $98.66 \%(221 / 224)$ \\
3 & 500 & $71 \%$ & 500 & $50 \%$ & $94.20 \%(211 / 224)$ \\
4 & 500 & $71 \%$ & 300 & $30 \%$ & $92.86 \%(208 / 224)$ \\
\hline \hline
\end{tabular}

The case study presented in this section is considered adequate to test and demonstrate the robustness and effectiveness of the proposed methodology. Different system scenarios and uncertainties, e.g., network topology, system loading levels, RES penetration and location, fault duration, etc., will produce new angle responses and may change the system GPs and the final CCMs deployment. However, the application of the method will remain unchanged, since the clustering parameters are statistically identified and the algorithm is entirely data-based with minimum heuristics involved. This is also the case for the subsequent stabilization procedure, which is based on the systematic ranking of events using the proposed acceleration indices. The definition of new scenarios and other uncertainties is straightforward using the proposed MC approach as described in Section II-A and Section III-B, C and D. Some of the system uncertainties have not been included in the presented case study, e.g., pre-fault generator and line outages, in order to facilitate simple and straightforward demonstration of the key aspects of the methodology. These uncertainties in pre-fault grid topology, in addition to the ones mentioned above (RES penetration, load levels, etc.), will also have an impact on the system oscillation patterns and hence should and can be easily included in the analysis using the proposed MC approach. In addition to considering the full range of uncertainties associated with power system operation, a techno-economic assessment of the solutions should also form an essential part of the development of practical solutions for grid stabilization.

Finally, it is also important to highlight that the precise information about the composition of GPs, as obtained in Table I, can be used for stabilization of other generator clusters apart from the critical one, as was the focus of the study case presented in this paper. These other clusters will oscillate at a lower rate than the critical group and can be acted upon in order to damp their corresponding oscillation 
modes. A critical generator for those other non-critical clusters could also be identified using the same proposed procedure so that any CCM deployment is optimized as well.

\section{CONCLUSIONS}

A procedure for the optimum deployment of CCMs for multi-machine transient stability improvement is proposed in this paper. It identifies first the critical generator oscillation patterns (Grouping Patterns-GP) by clustering probabilistically obtained rotor angle responses of a power system. The parameters of the Hierarchical Clustering (HC) algorithm used for grouping generator rotor angles were determined by statistically assessing the clustering results over an appropriate region of clustering parameters, almost eliminating the heavily heuristic criteria typically involved in this task. A new quality index, the Total Grouping Pattern ASW - TGPASW, based on a combination of the well-known Average Silhouette Width (ASW) and Gap Statistic (GS) indices to assess clustering quality, was proposed for this purpose. The efficacy of the proposed clustering method was demonstrated by identifying a reduced number of GPs that appropriately differentiate the multi-machine unstable oscillation patterns in a large test network, with realistic modelling of uncertainties, including those of RES. Furthermore, a comparison against HC with heuristically defined algorithm parameters demonstrated that the proposed method yields the minimum number of GPs and the maximum clustering quality indicators in all cases.

The identification of the GPs and their subsets of Critical Groups (CGs) facilitates the development of a systematic procedure for the stabilization of the network. The process consists of applying CCMs to the most Critical Generator (CGEN) to stabilize the most critical event per GP, both found with suitable defined acceleration indices, until full stabilization is achieved. Since only a few GPs for the system are identified, this process is fast and allows the critical generators for the entire system to be found easily. The final set of CCMs can be defined based on the results of the individual stabilization of the GPs. By identifying the CGENs, the locations for the most effective deployment of the CCMs are automatically defined. With the complete information about the composition of the GPs, CCMs could also be deployed optimally, following the same procedure, to stabilize other non-critical identified clusters of generators oscillating together, and such to damp other oscillation modes throughout the system.

To illustrate the proposed methodology, Switched Shunt Capacitors (SSHCs) were used as the CCM to stabilize the system. The choice of CCMs, however, is not relevant in the context of the aim of this study, as the proposed procedure can be performed with any non-disruptive CCM that is applied directly to the generators to be stabilized, such as Breaking Resistors, Switched Series Capacitors, Fast-Valving, series and shunt FACTS devices, Transient Excitation Boosting, etc. The proposed approach can also be potentially used with disruptive stabilization methods such as generation/load shedding and islanding schemes, and for on-line applications. Furthermore, the results obtained in the form of a probabilistic reduction of instability might be further used for evaluating corrective control in a risk-based fashion, comparing different types of corrective or even preventive control for a complete cost-effective stabilization of large uncertain power systems.

\section{REFERENCES}

[1] Task Force on Discrete Supplementary Controls of the Dynamic System Performance Working Group, "A Description of Discrete Supplementary Controls for Stability," IEEE Transactions on Power Apparatus and Systems, vol. PAS-97, no. 1, pp. 149-165, 1978.

[2] P. Kundur, Power System Stability and Control. New York, NY, USA: McGraw-Hill, 1994.

[3] J. D. Morales, P. N. Papadopoulos, and J. V. Milanović, "Feasibility of different corrective control options for the improvement of transient stability," in 2017 IEEE Manchester PowerTech, 2017, pp. 1-6.

[4] H. Jiang, J. Dorsey, T. Habetler, and K. v. Eckroth, "A cost effective generator brake for improved generator transient response," IEEE Transactions on Power Systems, vol. 9, no. 4, pp. 1840-1846, 1994.

[5] M. H. Ali, T. Murata, and J. Tamura, "Augmentation of transient stability by fuzzy-logic controlled braking resistor in multi-machine power system," in 2005 IEEE Russia Power Tech, 2005, pp. 1-7.

[6] K. K. Anaparthi, B. Chaudhuri, N. F. Thornhill, and B. C. Pal, "Coherency identification in power systems through principal component analysis," IEEE Transactions on Power Systems, vol. 20, no. 3, pp. 1658-1660, 2005.

[7] I. Kamwa, A. K. Pradhan, and G. Joos, "Automatic Segmentation of Large Power Systems Into Fuzzy Coherent Areas for Dynamic Vulnerability Assessment," IEEE Transactions on Power Systems, vol. 22, no. 4, pp. 1974-1985, 2007.

[8] R. Agrawal and D. Thukaram, "Identification of coherent synchronous generators in a Multi-Machine Power System using Support Vector Clustering," in 2011 International Conference on Power and Energy Systems, 2011, pp. 1-6.

[9] M. A. M. Ariff and B. C. Pal, "Coherency Identification in Interconnected Power System - An Independent Component Analysis Approach," IEEE Transactions on Power Systems, vol. 28, no. 2, pp. $1747-1755,2013$

[10] T. Guo and J. V. Milanović, "Identification of power system dynamic signature using hierarchical clustering," in 2014 IEEE PES General Meeting | Conference \& Exposition, 2014, pp. 1-5.

[11] T. Guo and J. V. Milanovic, "Online Identification of Power System Dynamic Signature Using PMU Measurements and Data Mining," IEEE Transactions on Power Systems, vol. 31, no. 3, pp. 1760-1768, 2016.

[12] P. N. Papadopoulos and J. V. Milanovic, "Probabilistic Framework for Transient Stability Assessment of Power Systems With High Penetration of Renewable Generation," IEEE Transactions on Power Systems, vol. 32, no. 4, pp. 3078-3088, 2017.

[13] P. N. Papadopoulos, T. Guo, and J. V. Milanović, "Probabilistic Framework for Online Identification of Dynamic Behavior of Power Systems With Renewable Generation," IEEE Transactions on Power Systems, vol. 33, no. 1, pp. 45-54, 2018.

[14] A. Semlyen, "Analysis of disturbance propagation in power systems based on a homogeneous dynamic model," IEEE Transactions on Power Apparatus and Systems, Article vol. PAS-93, no. 2, pp. 676-684, 1974.

[15] J. S. Thorp, C. E. Seyler, and A. G. Phadke, "Electromechanical wave propagation in large electric power systems," IEEE Transactions on Circuits and Systems I: Fundamental Theory and Applications, Article vol. 45, no. 6, pp. 614-622, 1998.

[16] M. Parashar, J. S. Thorp, and C. E. Seyler, "Continuum modeling of electromechanical dynamics in large-scale power systems," IEEE Transactions on Circuits and Systems I: Regular Papers, vol. 51, no. 9, pp. 1848-1858, 2004.

[17] T. Li, G. Ledwich, Y. Mishra, J. H. Chow, and A. Vahidnia, "Wave Aspect of Power System Transient Stability-Part I: Finite Approximation," IEEE Transactions on Power Systems, vol. 32, no. 4, pp. 2493-2500, 2017.

[18] T. Li, G. Ledwich, Y. Mishra, J. H. Chow, and A. Vahidnia, "Wave Aspect of Power System Transient Stability - Part II: Control Implications," IEEE Transactions on Power Systems, Article vol. 32, no. 4, pp. 2501-2508, 2017, Art no. 7707338 .

[19] J. D. Morales, J. V. Milanović, and P. N. Papadopoulos, "Analysis of Angular Threshold Criteria for Transient Instability Identification in 
Uncertain Power Systems," in 2019 IEEE Milan PowerTech, 2019, pp. $1-6$.

[20] B. S. Everitt, S. Landau, M. Leese, and D. Stahl, Cluster Analysis, 5th ed. Chichester, West Sussex, U.K.: John Wiley \& Sons Ltd, 2011.

[21] J. Han, M. Kamber, and J. Pei, Data Mining: Concepts and Techniques, 3rd ed. Waltham, MA, USA: Morgan Kaufmann, 2012.

[22] T. Hastie, R. Tibshirani, and J. Friedman, The Elements of Statistical Learning: Data Mining, Inference, and Prediction, 2nd ed. New York, USA: Springer, 2009.

[23] R. Tibshirani, G. Walther, and T. Hastie, "Estimating the number of clusters in a data set via the gap statistic," Journal of the Royal Statistical Society: Series B (Statistical Methodology), Article vol. 63, no. 2, p. 411, 2001.

[24] M. J. Baxter, Exploratory multivariate analysis in archaeology. Edinburgh: Edinburgh University Press, 1994.

[25] G. W. Milligan and M. C. Cooper, "An examination of procedures for determining the number of clusters in a data set," Psychometrika, Article vol. 50, no. 2, pp. 159-179, 1985.

[26] L. Kaufman and P. J. Rousseeuw, Finding Groups in Data: An Introduction to Cluster Analysis. Hoboken, NJ, USA: John Wiley \& Sons, Inc., 1990.

[27] G. Rogers, Power System Oscillations. Norwell, MA, USA: Kluwer Academic, 2000.

[28] B. Pal and B. Chaudhuri, Robust Control in Power Systems. New York, NY, USA: Springer, 2005.

[29] WECC Wind Power Plant Dynamic Modeling Guide. WECC Renewable Energy Modeling Task Force, Salt Lake City, UT, USA, 2014.

[30] WECC PV Power Plant Dynamic Modeling Guide. WECC Renewable Energy Modeling Task Force, Salt Lake City, UT, USA, 2014.

[31] Daily Load Curve Data, National Grid [Online] Available: http://www.nationalgrid.com/uk/electricity/market-operations-and-data/ data-explorer/

[32] M. Fan, V. Vittal, G. T. Heydt, and R. Ayyanar, "Probabilistic Power Flow Analysis With Generation Dispatch Including Photovoltaic Resources," IEEE Transactions on Power Systems, vol. 28, no. 2, pp. 1797-1805, 2013.

[33] M. Fan, V. Vittal, G. T. Heydt, and R. Ayyanar, "Probabilistic Power Flow Studies for Transmission Systems With Photovoltaic Generation Using Cumulants," IEEE Transactions on Power Systems, vol. 27, no. 4, pp. 2251-2261, 2012.

[34] S. Tao, Y. Ruoying, Z. Lingzhi, and G. Shan, "Power system probabilistic production simulation containing large-scale wind power and photovoltaic power," in 2013 IEEE PES Asia-Pacific Power and Energy Engineering Conference (APPEEC), 2013, pp. 1-6.

[35] R. Preece and J. V. Milanović, "Tuning of a Damping Controller for Multiterminal VSC-HVDC Grids Using the Probabilistic Collocation Method," IEEE Transactions on Power Delivery, vol. 29, no. 1, pp. 318326, 2014.

[36] J. P. Coelingh, A. J. M. v. Wijk, and A. A. M. Holtslag, "Analysis of wind speed observations over the North Sea," J. Wind Eng. Ind. Aerodyn., vol. 61, no. 1, pp. 51 - 69, 1996.

[37] Vestas Americas Inc., "V80-2.0 MW: Unsurpassed reliability and performance at high-wind sites in North America," 2011 [Online] Available: http://www.vestas.com

[38] T. Guo and J. V. Milanović, "Probabilistic Framework for Assessing the Accuracy of Data Mining Tool for Online Prediction of Transient Stability," IEEE Transactions on Power Systems, vol. 29, no. 1, pp. 377385, 2014.

[39] J. L. Rueda, D. G. Colome, and I. Erlich, "Assessment and Enhancement of Small Signal Stability Considering Uncertainties," IEEE Transactions on Power Systems, vol. 24, no. 1, pp. 198-207, 2009.

[40] J. D. Morales, P. N. Papadopoulos, and J. V. Milanović, "Statistical Assessment of the Impact of Renewable Energy Sources on Transient Stability," in 2018 IEEE PES Innovative Smart Grid Technologies Conference Europe (ISGT-Europe), 2018, pp. 1-6.
Juan D. Morales ( $\left.\mathrm{S}^{\prime} 16\right)$ received the B.Sc. degree (2007) and Dipl.Ing (2013) in electrical engineering from the Universidad Nacional de Ingeniería (UNI), Lima, Peru, and the M.Sc. degree in electrical power systems engineering from The University of Manchester, Manchester, U.K., in 2016, where he is currently pursuing the Ph.D. degree thanks to the M.Sc. Alumni $\mathrm{Ph}$.D. scholarship awarded by the same institution. From 2007 to 2015, he worked with Red de Energía del Perú (Peruvian TSO), holding positions in the planning, protection and regulation divisions of the system operations department. His current research interests are in the areas of probabilistic dynamic assessment and stabilization of uncertain power systems with increasing penetration of renewable energy sources.

Jovica V. Milanović (M'95-SM'98-F'10) received Dipl.Ing. and M.Sc. degrees from the University of Belgrade, Belgrade, Yugoslavia, a Ph.D. degree from the University of Newcastle, Newcastle, Australia, and a D.Sc. degree from the University of Manchester, Manchester, U.K., all in Electrical Engineering. He is currently a Professor of Electrical Power Engineering and a Deputy Head of Department of Electrical and Electronic Engineering at the University of Manchester, Manchester, U.K., and a Visiting Professor at the University of Novi Sad, Novi Sad, Serbia and University of Belgrade, Belgrade, Serbia. 